\title{
Expression of transient receptor potential channel 6 in cervical cancer
}

This article was published in the following Dove Press journal:

OncoTargets and Therapy

5 September 2012

Number of times this article has been viewed

Qi Wan'

\section{Ai Zheng' \\ Xiaojing Liu \\ Yali Chen' \\ Ling Han'}

'Department of Obstetric and Gynecology, West China Second University Hospital, ${ }^{2}$ Laboratory of Cardiovascular Diseases, Regenerative Medicine Research Center, West China Hospital, Sichuan University, Chengdu, China
Correspondence: Ai Zheng

Department of Obstetric and

Gynecology, West China Second

University Hospital, Sichuan University,

Chengdu 61004I, China

$\mathrm{Fax}+8602885422029$

Tel +8602885503349

Email zazheng716@hotmail.com

Xiaojing Liu

Laboratory of Cardiovascular Diseases,

Regenerative Medicine Research Center,

West China Hospital, Sichuan University,

Chengdu 61004I, China

Fax +8602885422029

$\mathrm{Tel}+8602885423049$

Email xiaojingliu67@yahoo.com
Background: Recent studies have reported that aberrant expression of transient receptor potential channel C6 (TRPC6) in a variety of human cancers is associated with aggressive behavior. However, the functional significance of TRPC6 in human cervical cancer is not known. This study was planned to detect whether TRPC6 is expressed in cervical cancer tissue and to evaluate the association between TPRC6 expression and clinicopathologic features.

Methods: Tissue samples were collected from the West China Second UNIV Hospital of Sichuan University. TRPC6 expression was detected by quantitative real-time reverse transcription polymerase chain reaction and Western blotting. TRPC6 expression was evaluated by immunohistochemistry analysis of 40 cervical cancer specimens, and correlations were sought between elevated expression of TRPC6 and clinicopathologic features.

Results: Increased expression of TRPC 6 was detected in 25 of the 40 cervical cancer samples. Positive cells found in cervical carcinomas were significantly increased in numbers compared with specimens without lymphovascular space invasion. Elevated expression of TRPC6 was neither related to International Federation of Gynecology and Obstetrics stage nor pelvic lymph metastases. Indeed, the clinicopathologic analysis indicated that overexpression of TRPC6 was significantly associated with lymphovascular space invasion.

Conclusion: These results indicate that elevated expression of TRPC6 might be associated with an aggressive cervical cancer phenotype.

Keywords: cervical cancer, transient receptor potential channel C6, overexpression, invasion

\section{Introduction}

Cervical cancer is one of the most common malignant cancers in females worldwide, and its incidence is high in the developing world. There are two major types of cervical cancer, ie, adenocarcinoma and squamous cell carcinoma, and the latter has especially high morbidity in China. Currently, it is widely accepted that cervical cancer can be caused by infection with certain strains of the human papillomavirus. ${ }^{1}$ Regimens of surgical resection, radiotherapy, and chemotherapy containing cisplatin improve survival rates among women with early-stage carcinoma. ${ }^{2}$ However, advanced carcinomas with lymphovascular space invasion (LVSI) still have a poor prognosis, and most patients eventually die from metastases or recurrence. Therefore, early markers of metastases and new regimes for tumor invasion are urgently needed.

Variable amounts of proteins were produced in cancer cells, and some of the proteins associated with cancer progression are involved in calcium homeostasis. Therefore, we focused on transient receptor potential canonical (TRPC), a nonselective 
cation channel, which is expressed in a variety of tissues and is involved in the proliferation of cancer cells. ${ }^{3-5}$

The transient receptor potential (TRP) superfamily is one of the largest ion channel families and consists of diverse groups of proteins. About 28 genes encode the TRP ion channel subunits in mammals. It comprises six subfamilies, including TRPC, which consists of seven proteins named TRPC 1 to 7 , and can be divided into three subgroups by sequence homology and functional similarities, ie, C1/C4/C5, $\mathrm{C} 3 / \mathrm{C} 6 / \mathrm{C} 7$, and $\mathrm{C} 2 .{ }^{6} \mathrm{TRPC} 6$ has been reported to be expressed in brain, lung, kidney, muscle and human platelets. ${ }^{7-11}$

Overexpression of this channel has also been found in $\mathrm{Ca}^{2+}$ signal generation involving many malignant tissues and cells, but the role and mechanism for this remain elusive. There is much evidence suggesting that TRPC6 is involved in the oncogenic process, and is selectively expressed in aggressive cancers and intimately involved in metastases. ${ }^{3-5,12,13}$ Recently, it has been reported that TRPC6 is also expressed in esophageal, gastric, breast, and prostate tissue, where elevated channel expression has been implicated in cancer of these organs. The role of TRPC6 in cell proliferation has been demonstrated in several types of cancer cells, using specific inhibitors, ie, SKF96365 or short interfering RNA blockade of TRPC6 channels, which suppress cancer cell proliferation. $3,5,13,14$ However, it is not clear how it works or even whether it works in cervical cancer.

It is clinically important to understand the role of ion channels in the development of cancer. Indeed, TRPC6 is strongly expressed in prostate and breast cancers. Moreover, its expression correlates with Gleason score and aggressiveness. On the basis of these observations, we hypothesized that this protein might be expressed in cervical carcinoma and an increased level of human TRPC6 protein might be associated with tumor invasion. To evaluate this hypothesis, we investigated the expression of TRPC6 protein in benign and malignant human cervical tissue, and correlations were sought between TRPC6 protein immunostaining and stage, LVSI, and pelvic lymph node metastases of the tumors.

\section{Materials and methods Samples}

Forty paraffin-embedded specimens and 12 paired fresh cervical samples were collected from the West China Second UNIV Hospital of Sichuan University (Sichuan, China) with ethics committee approval and consent from the patients. Tumor specimens were obtained from 40 cervical cancer patients, staged following International Federation of Gynecology and Obstetrics (FIGO) criteria, ie, 18 stage I tumors, 15 stage II tumors and 7 stage III tumors. All of the samples were squamous cell carcinomas. Normal tissue samples were collected from six healthy women who had undergone elective total hysterectomies due to benign uterine disease (leiomyoma). These samples did not contain any histologic evidence of cervical intraepithelial neoplasia or invasive carcinoma. Clinicopathologic parameters, including age at time of surgery, tumor grade, lymph node status, and presence of LVSI, were extracted from the histopathologic examination results.

\section{Total RNA extraction}

All the samples used were analyzed by two experienced pathologists to ensure that they were correctly identified as either benign cervical tissue or cervical cancer. Total tissue RNA was extracted using Trizol reagent (Invitrogen, Carlsbad, CA), and cDNA was synthesized from total RNA using oligodT primers (Invitrogen). RNA integrity was confirmed by electrophoresis on $1 \%$ agarose gels stained with ethidium bromide.

\section{Quantitative reverse-transcription PCR}

Quantitative reverse-transcription polymerase chain reaction (PCR) was utilized to compare the relative amounts of TRPC6 in the fresh cervical tissue biopsy samples and was carried out on a Bio-Rad System (CFX96, Hercules, CA). For each reaction, cDNA was added to $20 \mu \mathrm{L}$ of reaction mixture containing $10 \mu \mathrm{L}$ of SYBR Green PCR Master Mix (Toyobo, Osaka, Japan) and $300 \mathrm{nM}$ primers (Invitrogen). For quantification, the target sequence was normalized to GAPDH mRNA levels. PCR was performed under the following conditions: $95^{\circ} \mathrm{C}$ for 30 seconds, $57^{\circ} \mathrm{C}$ for 15 seconds, and $72^{\circ} \mathrm{C}$ for 30 seconds for 40 cycles. Heating for 2 minutes at $95^{\circ} \mathrm{C}$ preceded the cycles. We quantified the results using the comparative CT method. PCR products were analyzed by electrophoresis with $1.5 \%$ agarose gel and visualized by ethidium bromide staining. Primer sequences used for amplification were as follows: TRPC6 (product size $152 \mathrm{bp}$ ) upstream primer, 5'-GCCAATGAGCATCTGGAAAT-3'; downstream primer, 5'-AACCTCTTGCCTTCAGCAAA-3'; GAPDH (product size 200 bp) upstream primer, 5'-AACTGCTTAGCACCCCTGGC-3'; downstream primer, 5'-ATGACCTTGCCCACAGCCTT-3'.

\section{Western blotting}

Cervical tissue proteins were extracted using ice-cold lysis buffer containing a protease inhibitor cocktail (Roche, Meylan, France), and the proteins in the supernatant were 
quantified using the bicinchoninic acid method (Pierce, Rockford, IL). Fifty micrograms of protein were separated per lane by $10 \%$ sodium dodecyl sulfate-polyacrylamide gel electrophoresis. After electrophoretic transfer of the proteins to a polyvinylidene difluoride membrane (Millipore, Bedfordshire, UK) using a MiniProtein III system (Bio-Rad), immunoblotting was performed using rabbit polyclonal primary anti-TRPC6 antibody (diluted 1:2000, Abcam, Cambridge, UK) and primary anti- $\beta$-actin (diluted 1:4000, Santa Cruz Biotechnologies, Santa Cruz, CA), and developed in an enhanced chemiluminescence system (Pierce) using specific peroxidase-conjugated anti-IgG secondary antibodies (1:4000 dilution, Santa Cruz). For quantification purposes, densitometric measurements were performed using the Quantity One image analysis software for Windows (Bio-Rad). All TRPC6 values were normalized to $\beta$-actin levels.

\section{Immunohistochemistry}

Paraffin sections were cut and mounted on glass slides, and $5 \mu \mathrm{m}$ sections from formalin-fixed and paraffin-embedded specimens were deparaffinized using xylene and rehydrated in graded ethanol. Samples were then preincubated with 3\% $\mathrm{H}_{2} \mathrm{O}_{2}$ to eliminate endogenous peroxidase activity. Antigen retrieval was achieved by heating the sections (for 2 minutes to $\left.100^{\circ} \mathrm{C}\right)$ in citric acid buffer $(0.01 \mathrm{~mol} / \mathrm{L}, \mathrm{pH} 6.0)$. Sections were incubated at $4{ }^{\circ} \mathrm{C}$ overnight in a 1:800 dilution of the rabbit polyclonal primary anti-TRPC6 antibody (Abcam). After three washes, goat anti-rabbit horseradish peroxidaseconjugated antibody (Envision detection kit, Gene-Tech, Shanghai, China) was applied for one hour. Sections were developed using a peroxidase substrate DAB kit (GeneTech) and counterstained using hematoxylin. Sections were subsequently dehydrolyzed before they were mounted on coverslips. Images were collected using an Eclipse TE2000-U microscope system (Nikon, Tokyo, Japan). The primary antibody was replaced with phosphate-buffered saline in the control experiments. All of the sections diagnosed as cervical squamous cell carcinoma were evaluated using conventional hematoxylin and eosin staining.

TRPC6 staining in immunohistochemistry was evaluated by the percentage of positive cells: $0 \%-10 \%$ of positive tumor cells; $10 \%-25 \%$ of positive tumor cells; $25 \%-50 \%$ of positive tumor cells; and $>50 \%$ of positive tumor cells. Tumors were evaluated by random observation of five fields on every section by two investigators. The mean score served as the tumor TRPC6 expression score. Overexpression was defined as $>25 \%$ of positive cell staining in the study without consideration of the intensity of staining.

\section{Statistical analysis}

All the experiments were performed at least three times. Contingency table, $\chi^{2}$ analyses, and group means were compared by one-way analysis of variance using the Statistical Package for Social Sciences software version 17.0 (SPSS Inc, Chicago, IL). Differences resulting in $P<0.05$ were considered to be statistically significant.

\section{Results \\ Increased TRPC6 expression in human epithelial cervical cancer}

To determine whether TRPC6 mRNA expression differs in benign and malignant cervical tissue, tissue mRNA extracted from different patients was analyzed using quantitative PCR. The results presented are the means of the measurements for 12 individual specimens. From our study, it can be seen that both benign and diseased tissue expressed TRPC6 at the genetic level. TPRC6 expression was significantly higher in malignant tissue than in benign cervical tissue $(P<0.05$, Figure 1).

\section{Expression of TRPC6 in benign and malignant human cervical tissues}

To our knowledge, no data are available on expression of TRPC6 in human cervical carcinoma tissue. We verified TRPC6 expression in tissues freshly collected after surgery. Transcripts for TRPC6 were expressed in these tissues, and expression was clearly higher in cancer tissue than in benign tissue by semiquantitative Western blotting $(P<0.05$, Figure 2). Analysis of TRPC6 protein expression showed results consistent with quantitative PCR.

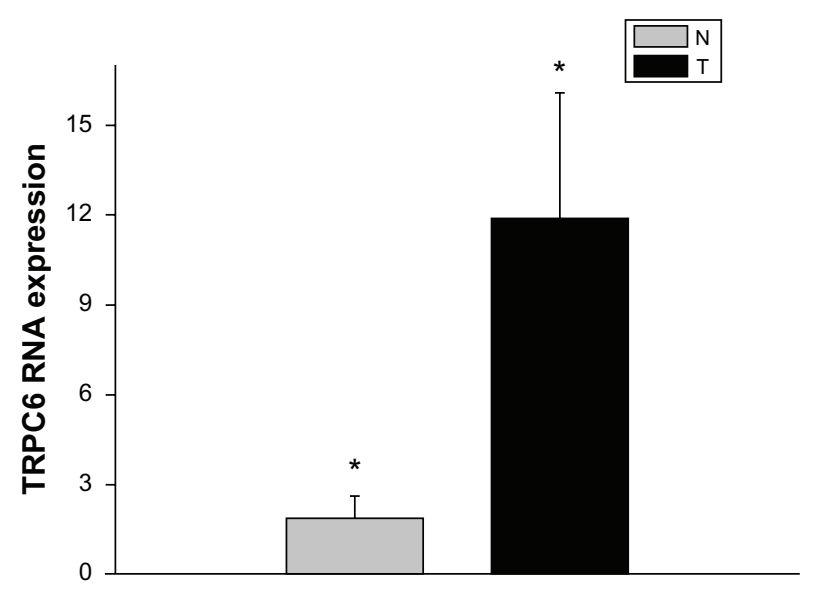

Figure I mRNA expression of TRPC6 in cervical cancer compared with benign cervical tissue.

Notes: Data are represented as means of duplicate determinations of six normal cervical samples obtained from six patients with benign uterine diseases $(\mathrm{N})$ and of six cervical cancer samples $(\mathrm{T})$. $* \mathrm{P}<0.05$.

Abbreviation: TRPC6, transient receptor potential channel C6. 


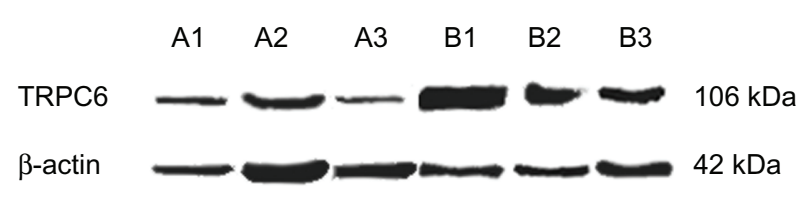

Figure 2 Representative Western blotting of three determinations (mean \pm standard error of the mean, bottom). $P<0.05$ versus $\mathrm{Al}-3$.

Abbreviations: $\mathrm{Al}-3$, benign cervical tissue protein; $\mathrm{BI}-3$, cervical cancer tissue protein; K Da, Kilodalton.

\section{Association of TRPC6 protein expression and LVSI in cervical carcinoma}

In the present study, 40 cervical carcinoma specimens in paraffin sections were analyzed using immunohistochemistry in order to determine expression of TRPC6. TRPC6 protein was localized to the plasma membrane, and only the channels expressed in the zones affected by carcinoma were included. Consequently, overexpression of the protein was detected in 10 of 18 stage I tumors (55.6\%), eight of 15 stage II tumors $(53.3 \%)$, and seven of seven stage III tumors (100\%). A Chisquare test showed that TRPC6 expression did not significantly correlate with clinicopathologic factors, including age, FIGO stage, and lymph node metastatic status $(P>0.05)$. TRPC6 expression was significantly associated with LVSI of cervical carcinoma $(P<0.05$, Table 1$)$. Staining was less intense when the primary antibody was substituted with phosphate-buffered saline (Figure 4). Although more than $50 \%$ of tumor cells expressing TRPC6 protein was noted in $11.1 \%$ of stage I, $26.6 \%$ of stage II, and $42.9 \%$ of stage III tumors, no significant difference was found in FIGO stage $(P>0.05)$.

\section{Discussion}

In our study, we investigated the association between expression of TRPC6 in squamous cell carcinoma and LVSI. We initially

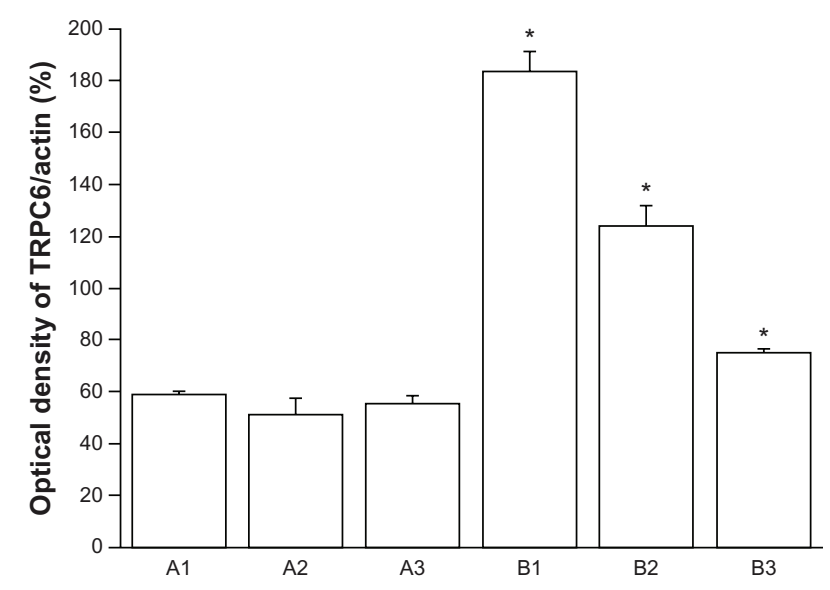

Figure 3 Representative Western blotting of three determinations (mean \pm standard error of the mean, bottom).

Abbreviations: $\mathrm{Al}-3$, benign cervical tissue protein; $\mathrm{BI}-3$, cervical cancer tissue protein; K Da, Kilodalton.

Note: $* P<0.05$ versus Al-3.
Table I Comparison of TRPC6 expression to tumor characterics on 40 patients using $\chi 2$ analysis

\begin{tabular}{|c|c|c|c|c|c|c|}
\hline & \multirow[t]{2}{*}{$\mathbf{n}$} & \multicolumn{4}{|c|}{ TRPC6 expression (positive cells ratio) } & \multirow[t]{2}{*}{$\mathbf{X}^{2}$} \\
\hline & & $0 \%-10 \%$ & $10 \%-25 \%$ & $25 \%-50 \%$ & $>\mathbf{5 0} \%$ & \\
\hline \multicolumn{7}{|l|}{ Age } \\
\hline$>40$ & 27 & 2 & 7 & 11 & 7 & \\
\hline$<40$ & 13 & 3 & 3 & 5 & 2 & 2.197 \\
\hline \multicolumn{7}{|c|}{ Pathological stage } \\
\hline I & 18 & 3 & 5 & 8 & 2 & \\
\hline II & 15 & 2 & 5 & 4 & 4 & 7.068 \\
\hline III & 7 & 0 & 0 & 4 & 3 & 7.068 \\
\hline \multicolumn{7}{|c|}{ Pelvic lymph metastatis } \\
\hline+ & 17 & 2 & 4 & 8 & 3 & \\
\hline- & 23 & 3 & 6 & 8 & 6 & 0.716 \\
\hline \multicolumn{7}{|c|}{ Lymphovascular space invasion } \\
\hline+ & 20 & 0 & 3 & 11 & 6 & \\
\hline- & 20 & 5 & 7 & 5 & 3 & 9.85 \\
\hline
\end{tabular}

Abbreviations: TRPC6, transient receptor potential channel C6.

assessed the expression of TRPC6 channels in benign and cervical cancer tissue samples removed at surgery. Increased expression of TRPC6 in patients with malignant cervical tumors was found. Second, results from the Western blotting and quantitative PCR were consistent. Expression of TRPC6 was shown in all of the tissues, while overexpression was only found in malignant tissues. Third, on the basis of results from histologic sections of cervical cancer tissue, it can be concluded that LVSI results in increased numbers of TRPC6 positive cells. About $25 \%-50 \%$ of positive cells were found in 11 of $16(68.75 \%)$ LVSI samples, and more than $50 \%$ were shown in six of nine (66.7\%) LVSI samples. There was a significant association between LVSI and TRPC6 positive cells $(P<0.05)$.

LVSI, including lymphatic vessel invasion and blood vessel invasion, is an important factor in the metastatic potential of cervical carcinoma. Therefore, it plays a predictive role in lymph node metastases and ovarian metastases. ${ }^{15-18}$ Patients with LVSI may have completely different clinical outcomes. This study shows that overexpression of TRPC6 is associated with malignant tissue and LVSI, and therefore it may be a useful biomarker for metastases in cervical cancer. Determination of levels of this protein may help to guide adjuvant therapies, such as radiotherapy and/or chemotherapy, for an individual patient with cervical cancer postoperatively. Otherwise, blockers targeted toward these channels may provide promising new therapeutic solutions to protect against development of tumors.

Indeed, evidence from existing research has shown that blocking TRPC6 using short interfering RNA inhibition of calcium ion influx induces cell cycle arrest in the G2/M phase and growth retardation in AGS and MKN45 gastric cancer 


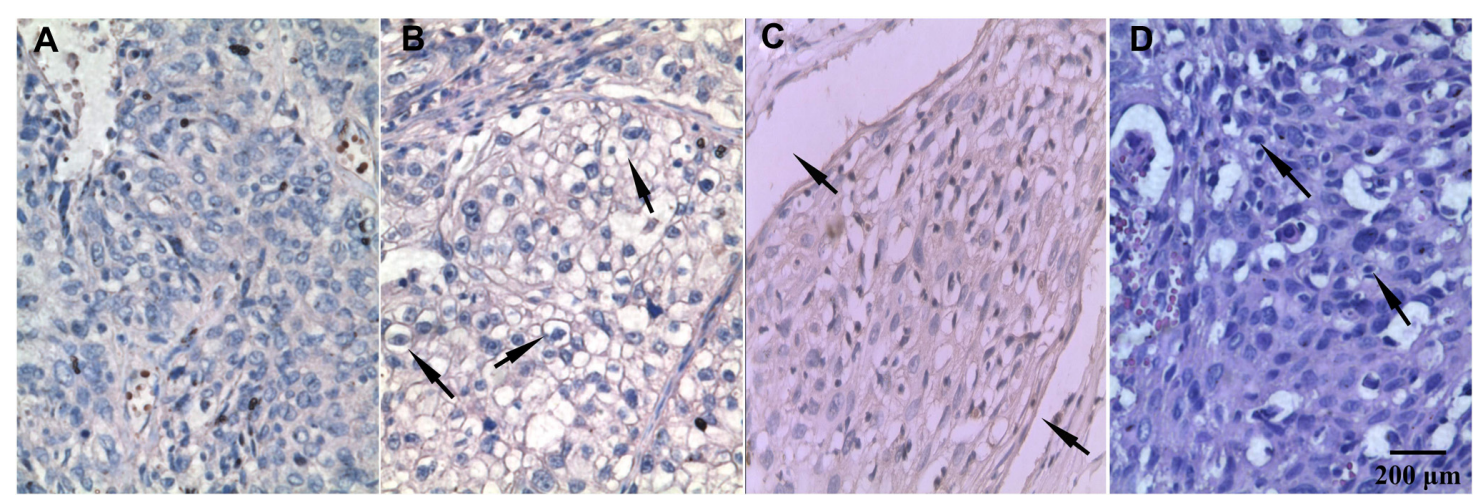

Figure 4 Immunohistochemistry staining of TRPC6 protein expressed in human cervical carcinoma tissues (I00X). (A) There was little to no cell membrane staining when the primary antibody was substituted with phosphate-buffered saline. (B) More than $50 \%$ cells stained positive for TRPC6, cell membrane staining was seen at the arrow. (C) TRPC6-stained sample with vascular wall invasion, which was indicated at the arrow. (D) Hematoxylin and eosin staining sample with nuclear atypia at the arrow.

Abbreviation: TRPC6, transient receptor potential channel C6.

cell lines. ${ }^{3}$ Similar results were also observed in esophageal and ovarian cancer cell lines. Moreover, inhibition of TRPC6 or TRPC3 can suppress tumor formation in nude mice. ${ }^{19,20}$ It is thought to regulate by capacitative $\mathrm{Ca}^{2+}$ entry. ${ }^{19,20} \mathrm{Ca}^{2+}$ signaling is important for proliferation and survival of cancer cells ${ }^{21}$ and TRPC6-mediated $\mathrm{Ca}^{2+}$ influx also increases RhoA activity, which may regulate cell migration. ${ }^{22}$ Therefore, we have reason to speculate that TRPC6, which is mainly permeable to $\mathrm{Ca}^{2+}$, may play some role in the development of cancer. TRPC6 and TRPC 3 are nonselective cation channels, and are directly activated by diacylglycerol through a mechanism independent of protein kinase $\mathrm{C} .{ }^{23}$ Increases in $\mathrm{Ca}^{2+}$ influx not only control gene expression, cell cycle progression, and apoptosis, but also modify cell growth and differentiation. ${ }^{24,25}$ However, overexpression of TRPC6 was not observed in esophageal and HEK293 cell proliferation or an alteration in $\mathrm{G} 2 / \mathrm{M}$ phase transition. ${ }^{19}$ Based on these results, it is suggested that $\mathrm{Ca}^{2+}$ regulated by TRPC 6 channels might be involved in tumorigenesis, but it does not control the growth of tumor cells.

Although previous studies have shown that positive TRPC6 expression predicts a poor clinical prognosis in breast and prostate cancer, our study, which used immunohistochemistry to detect overexpression TRPC6 protein in 25 squamous cell carcinoma tissue slices, did not indicate that TRPC6 expression was significantly correlated with FIGO stage in cervical cancer $(P>0.05)$.

Radiotherapy and chemotherapy is conventionally recommended instead of surgery for patients with stage III and IV cervical cancer. Therefore, it is difficult to find tissue samples at an advanced clinical stage, and this is the reason for the small sample size in our study. It should be pointed out that tumor cells in the $\mathrm{G} 2 / \mathrm{M}$ phase are most sensitive to radiation, ${ }^{26}$ with other studies reporting that downregulation of TRPC6 contributes to G2/M cell cycle transition of cancer cells. We propose that FIGO III stage cervical cancer with upregulated expression of TRPC6 is likely to be more sensitive to radiotherapy.

In our experiment, we only detected expression of TRPC6 and the relationship between its expression and the prognosis of cervical cancer. The mechanism of increased TRPC6 channel expression in cervical cancer remains unknown. We postulate that the development of cervical carcinoma via increased TRPC6 channel expression might be associated with exchange of calcium ions. Further research is required to clarify the role of TRPC6 in this process.

\section{Acknowledgment}

This work was supported by grants from the Sichuan Science and Technology Agency publication (NO.2011FZ0041) and National Natural Science Foundation of China (No.30870598, No.11072163).

\section{Disclosure}

The authors report no conflicts of interest in this work.

\section{References}

1. Walboomers JM, Jacobs MV, Manos MM, et al. Human papillomavirus is a necessary cause of invasive cervical cancer worldwide. J Pathol. 1999;189(1):12-19.

2. [No authors listed]. Correction: concurrent cisplatin-based radiotherapy and chemotherapy for locally advanced cervical cancer. $N$ Engl J Med. 1999;341(9):708.

3. Cai R, Ding X, Zhou K, et al. Blockade of TRPC6 channels induced $\mathrm{G} 2 / \mathrm{M}$ phase arrest and suppressed growth in human gastric cancer cells. Int J Cancer. 2009;125(10):2281-2287.

4. El Boustany C, Bidaux G, Enfissi A, Delcourt P, Prevarskaya N, Capiod T. Capacitative calcium entry and transient receptor potential canonical 6 expression control human hepatoma cell proliferation. Hepatology. 2008;47(6):2068-2077. 
5. Guilbert A, Dhennin-Duthille I, Hiani YE, et al. Expression of TRPC6 channels in human epithelial breast cancer cells. BMC Cancer. 2008;8:125.

6. Bodding M. TRP proteins and cancer. Cell Signal. 2007;19(3): 617-624.

7. Bardell TK, Barker EL. Activation of TRPC6 channels promotes endocannabinoid biosynthesis in neuronal CAD cells. Neurochem Int. 2010;57(1):76-83.

8. Zhang MF, Liu XR, Yang N, Lin MJ. TRPC6 mediates the enhancements of pulmonary arterial tone and intracellular $\mathrm{Ca}^{2+}$ concentration of pulmonary arterial smooth muscle cells in pulmonary hypertension rats. Sheng Li Xue Bao. 2010;62(1):55-62. Chinese.

9. Moller CC, Wei C, Altintas MM, et al. Induction of TRPC6 channel in acquired forms of proteinuric kidney disease. J Am Soc Nephrol. 2007;18(1):29-36.

10. Jung S, Strotmann R, Schultz G, Plant TD. TRPC6 is a candidate channel involved in receptor-stimulated cation currents in A7r5 smooth muscle cells. Am J Physiol Cell Physiol. 2002;282(2):C347-C359.

11. Hassock SR, Zhu MX, Trost C, Flockerzi V, Authi KS. Expression and role of TRPC proteins in human platelets: evidence that TRPC6 forms the store-independent calcium entry channel. Blood. 2002;100(8): 2801-2811.

12. Chigurupati S, Venkataraman R, Barrera D, et al. Receptor channel TRPC6 is a key mediator of Notch-driven glioblastoma growth and invasiveness. Cancer Res. 2010;70(1):418-427.

13. Yue D, Wang Y, Xiao JY, Wang P, Ren CS. Expression of TRPC6 in benign and malignant human prostate tissues. Asian J Androl. 2009;11(5):541-547.

14. Ding X, He Z, Shi Y, Wang Q, Wang Y. Targeting TRPC6 channels in oesophageal carcinoma growth. Expert Opin Ther Targets. 2010;14(5): 513-527.

15. Sakuragi N, Takeda N, Hareyama H, et al. A multivariate analysis of blood vessel and lymph vessel invasion as predictors of ovarian and lymph node metastases in patients with cervical carcinoma. Cancer. 2000;88(11):2578-2583.
16. Wu HS, Yen MS, Lai CR, Ng HT. Ovarian metastasis from cervical carcinoma. Int J Gynaecol Obstet. 1997;57(2):173-178.

17. Morice P, Piovesan P, Rey A, et al. Prognostic value of lymphovascular space invasion determined with hematoxylin-eosin staining in early stage cervical carcinoma: results of a multivariate analysis. Ann Oncol. 2003;14(10):1511-1517.

18. Silva AL, Reis FM, Traiman P, Predrosa MS, Miranda D, Triginelli SA. Clinicopathological features influencing pelvic lymph node metastasis and vaginal and parametrial involvement in patients with carcinoma of the cervix. Gynecol Obstet Invest. 2005;59(2):92-96.

19. Shi Y, Ding X, He ZH, Zhou KC, Wang Q, Wang YZ. Critical role of TRPC6 channels in G2 phase transition and the development of human oesophageal cancer. Gut. 2009;58(11):1443-1450.

20. Yang SL, Cao Q, Zhou KC, Feng YJ, Wang YZ. Transient receptor potential channel $\mathrm{C} 3$ contributes to the progression of human ovarian cancer. Oncogene. 2009;28(10):1320-1328.

21. Roderick HL, Cook SJ. $\mathrm{Ca}^{2+}$ signalling checkpoints in cancer: remodelling $\mathrm{Ca}^{2+}$ for cancer cell proliferation and survival. Nat Rev Cancer. 2008;8(5):361-375.

22. Tian D, Jacobo SM, Billing D, et al. Antagonistic regulation of actin dynamics and cell motility by TRPC5 and TRPC6 channels. Sci Signal. 2010;3(145):ra77.

23. Hofmann T, Obukhov AG, Schaefer M, Harteneck C, Gudermann T, Schultz G. Direct activation of human TRPC6 and TRPC3 channels by diacylglycerol. Nature. 1999;397(6716):259-263.

24. Clapham DE. Calcium signaling. Cell. 2007;131(6):1047-1058.

25. Berridge MJ, Bootman MD, Lipp P. Calcium - a life and death signal. Nature. 1998;395(6703):645-648.

26. Lobrich M, Jeggo PA. The impact of a negligent G2/M checkpoint on genomic instability and cancer induction. Nat Rev Cancer. 2007;7(11): 861-869.
OncoTargets and Therapy

\section{Publish your work in this journal}

OncoTargets and Therapy is an international, peer-reviewed, open access journal focusing on the pathological basis of all cancers, potential targets for therapy and treatment protocols employed to improve the management of cancer patients. The journal also focuses on the impact of management programs and new therapeutic agents and protocols on

\section{Dovepress}

patient perspectives such as quality of life, adherence and satisfaction The manuscript management system is completely online and includes a very quick and fair peer-review system, which is all easy to use. Visit http://www.dovepress.com/testimonials.php to read real quotes from published authors. 